\title{
Recent Updates in Antimicrobial Stewardship in Outpatient Parenteral Antimicrobial Therapy
}

\author{
Monica V. Mahoney ${ }^{1}\left[\right.$ - Lindsey M. Childs-Kean ${ }^{2} \cdot$ Parisa Khan $^{3} \cdot$ Christina G. Rivera $^{4} \cdot$ Ryan W. Stevens $^{4}$. \\ Keenan L. Ryan ${ }^{5}$
}

Accepted: 22 October 2021 / Published online: 9 November 2021

(c) The Author(s), under exclusive licence to Springer Science+Business Media, LLC, part of Springer Nature 2021

\begin{abstract}
Purpose of Review Antimicrobial stewardship within acute care is common and has been expanding to outpatient areas. Some inpatient antimicrobial stewardship tactics apply to outpatient parenteral antimicrobial therapy (OPAT) and complex outpatient antimicrobial therapy (COpAT) management, but differences do exist.

Recent Findings OPAT/COpAT is a growing area of practice and research with its own unique considerations for antimicrobial stewardship. Potential ideas for antimicrobial stewardship in the OPAT/COpAT setting include redesigning the regimen to COpAT instead of OPAT, ensuring the use of the shortest effective duration of antimicrobial therapy; using antimicrobials dosed less frequently, such as long-acting glycopeptides; optimizing antimicrobial susceptibility testing reporting for common OPAT/COpAT drugs; and establishing routine laboratory and safety monitoring. Future consensus is needed to determine validated OPAT program metrics and outcomes.

Summary As more focus is placed on outpatient antimicrobial stewardship, clinicians practicing in OPAT should publish more data regarding OPAT program methods and outcomes as they relate to antimicrobial stewardship. These can involve patient clinical outcomes, OPAT readmission rates, OPAT therapy completion, and central line-related complications.
\end{abstract}

Keywords Antimicrobial stewardship $\cdot$ Home care $\cdot$ Outpatient parenteral antimicrobial therapy $\cdot$ OPAT

\section{Introduction}

Outpatient parenteral antimicrobial therapy (OPAT) is the administration of intravenous (IV) antimicrobials outside of the acute care hospital setting for at least 2 doses without

This article is part of the Topical Collection on Antimicrobial Development and Drug Resistance

Monica V. Mahoney

mmahoney@bidmc.harvard.edu

Lindsey M. Childs-Kean

Lchilds-kean@cop.ufl.edu

Parisa Khan

Parisa.khan@va.gov

Christina G. Rivera

Rivera.christina@mayo.edu

Ryan W. Stevens

stevens.ryan@mayo.edu

Keenan L. Ryan

Kryan2@salud.unm.edu intervening hospitalization [1]. OPAT is an alternative to inpatient care administered in a variety of models including at infusion centers or ambulatory care clinics, at home with nursing services or caregiver(s), and in skilled nursing facilities [2]. Many infections are treated via OPAT, including bone and joint, skin and soft tissue, pulmonary, central nervous system, intra-abdominal, catheter-associated, urogenital,

1 Beth Israel Deaconess Medical Center, Boston, MA, USA

2 Department of Pharmacotherapy and Translational Research, University of Florida College of Pharmacy, Gainesville, FL, USA

3 Michael E. DeBakey VA Medical Center, Houston, TX, USA

4 Department of Pharmacy, Mayo Clinic, Rochester, MN, USA

5 Inpatient Pharmacy Department, University of New Mexico Hospital, Albuquerque, NM, USA 
cardiac device related, and endocarditis. Treatment durations generally range $2-8$ weeks. Clinicians may choose oral antimicrobial(s) with adequate bioavailability and infection site penetration as an OPAT alternative. Oral antimicrobials used for extended periods of time or that require outpatient monitoring have been termed complex outpatient antimicro-

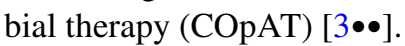

While long term, often broad-spectrum, antimicrobials appear ripe for stewardship interventions, inpatient methodologies do not directly translate to OPAT/COpAT. Traditional stewardship cornerstones such as prospective audits with intervention and feedback, formulary restriction, and preauthorization can be challenging to apply to OPAT/COpAT [4]. Prospective audits can be rapidly implemented in data-rich inpatient environments using real-time antimicrobial orders and administration records. However, these are not available for outpatients in the home and limited in skilled facilities/infusion centers [5]. Capture of antimicrobial days of therapy (DOT) and other longitudinal antimicrobial usage metrics face a similar data barrier. An additional challenge is that OPAT patients' settings can change throughout the therapy course. Outside of single payer systems, antimicrobial formulary restrictions and preauthorizations are difficult to apply as individual patient's insurance companies generally maintain formulary control. Lastly, drug dispensing may be externally controlled by for-profit infusion companies; thus, product-based stewardship cost containment initiatives (e.g., batching, extending infusions, contracting purchases prices, etc.) are limited or non-existent.

OPAT/COpAT is commonly definitive therapy administered by patients or caregivers; therefore, the OPAT regimen design prioritizes ease of administration, drug consolidation, safety, and tolerability to optimize patient or caregiver adherence along with antimicrobial spectrum and pharmacokinetics/pharmacodynamics (PK/PD). A narrow spectrum of activity is often sacrificed to promote adherence, which should be taken into account within OPAT/COpAT stewardship methods. While inpatient stewardship models do not neatly fit into OPAT/COpAT, this creates opportunities for new and re-invented stewardship methods.

In this paper, we review recent advances in OPAT literature, specifically with antimicrobial stewardship objectives, with a focus on publications within the last five years.

\section{Regimen Redesign from OPAT to COpAT}

A critical target for antimicrobial stewardship efforts in the context of OPAT is consideration for changing an OPAT regimen to COpAT [6]. Transitioning IV antimicrobials into oral regimens may occur at various time points and in varied fashions throughout the OPAT care process. This may include transition at the time of discharge, following initial discharge on OPAT, or the use of a mixed regimen containing both intravenously and orally administered antibiotics. Specifically, employing the use of COpAT at the time of discharge avoids the need for peripherally inserted central catheters (PICCs) which are associated with numerous potential complications including bloodstream infection, deep vein thrombosis, insertion site redness or discomfort, accidental removal, and/or catheter occlusion [7, 8]. Estimated complication rates vary; however, one study demonstrated that $61.4 \%$ of outpatients self-reported at least one PICCrelated complication [7]. The economic impact of avoiding PICCs should also be considered, given cost estimates for placement alone ranging from $\$ 158.40$ to $\$ 690.00$ per patient [9]. COpAT can also negate OPAT requirements for home health, frequent infusion therapy clinic visits, or even entirely avoid OPAT-related admission to a skilled facility. During the COVID-19 pandemic where minimization of exposure risk was critical, use of COpAT in place of OPAT was of particular interest for these reasons [10].

Beyond the minimization of line placement and healthcare exposure, COpAT is accompanied by other significant benefits. A study of pediatric patients comparing economic and patient-related benefits of those receiving COpAT with those receiving OPAT found COpAT was associated with significantly lower direct costs of therapy, less missed school for children, less missed work for caregivers, a higher level of caregiver comfort with the planned regimen, and better caregiver quality of life [11]. Multiple studies have evaluated the impact of the use of oral antimicrobials or implementation of protocols allowing for the use of oral antibiotics in the treatment of bone and joint infections [12•,13]. One such study evaluated the economic impact of transition to COpAT in eligible patients with bone and joint infections demonstrated a 19.5 day reduction in IV antimicrobial administration which was associated with $£ 1234$ (\$1746.48) in cost savings per patient. Notably, in this study, those who transitioned to COpAT did so after a median of 17 days of OPAT, thereby demonstrating the value of ambulatory OPAT to COpAT transition [12•].

When looking to implement the use of COpAT, several important factors should be considered. As opposed to simply changing the same antimicrobial from IV to oral formulation, creation of a COpAT regimen usually requires modification of an IV regimen to different drug(s) and/or drug classes. Therefore, COpAT selection must be accompanied by careful evaluation of patient factors that may complicate outcomes (e.g., gastrointestinal malabsorption potential, non-adherence) or misalign with study protocols (e.g., organisms not included, failure to meet "clinical stability" definitions). Additionally, COpAT must provide sufficient coverage of suspected or confirmed pathogens, and ideally should have high oral bioavailability and achieve 
adequate tissue penetration at the infection site. It should be clearly stated that COpAT is not void of complications. Oral antimicrobials have been associated with significant rates of discontinuation, especially when administered at high doses and for long durations such as during prosthetic joint infections [14]. Patients receiving COpAT are recommended to have close follow-up for medication adherence, antimicrobial tolerance, and clinical improvement. Therefore, the practice of instituting and monitoring COpAT is best performed by those with expertise in infectious diseases (ID), and represents an opportunity for OPAT teams to identify COpAT candidates, aid in agent selection, and provide close follow-up $[3 \bullet \bullet$.

\section{Antimicrobial Duration}

Optimizing duration of antimicrobial therapy is a key tenant of antimicrobial stewardship [15]. There has been a significant push to decrease the duration of therapy for many common infectious etiologies. In some cases, a short duration may preclude patients from even needing OPAT/ COpAT $[16,17]$. Notable examples of possible shortened durations for infections treated in the OPAT/COpAT setting include but are not limited to: diabetic foot infections with osteomyelitis (3 vs. 6 weeks) [18], septic arthritis (2 vs. 4 weeks) [19], and vertebral osteomyelitis (6 vs. 12 weeks) [20]. It should be noted that many of these trials evaluated duration of treatment with optimum source control. Given the diversity of patient presentations and care provided prior to hospital discharge, a short duration should not be a guarantee for all patients but a decision that is based on the clinical evolution of the patient. For example, increases in age, C-reactive protein, and more frequent OPAT clinic monitoring were all associated with longer durations of IV antibiotics for skin and soft-tissue infections within a single English teaching hospital [21]. The ideal approach remains to limit antibiotic exposure to the shortest effective duration in order to eradicate an infection, which cannot always be projected at the start of therapy.

\section{Microbiology Collaboration and Coordination}

Significant effort has been invested to optimize the collaboration between the microbiology laboratory and antimicrobial stewardship programs. One such intervention has been the use of antimicrobial cascade reporting (CR). By selectively reporting susceptibility based on specific results, $\mathrm{CR}$ has nudged providers into de-escalating antimicrobial treatments without requiring significant dedicated time from the antimicrobial stewardship program. Cascade report- ing has been associated with a significant increase in deescalation without increasing length of stay, Clostridioides difficile rates, or mortality [22•, 23, 24]. While CR is a high yield intervention, it should be implemented thoughtfully to ensure there are minimal downstream repercussions for OPAT/COpAT patients. It is not uncommon for OPAT/ COpAT to require adjustment for a variety of reasons. For example, a patient with Staphylococcus aureus bacteremia and osteomyelitis may be de-escalated to oral antibiotics when clinically appropriate. Susceptibilities for this isolate will likely be based on the blood culture which may not report oral antimicrobial susceptibilities. Conversely, a patient may need a broader antimicrobial agent secondary to an adverse event or isolation of an organism with a different resistance profile [25]. It is common for isolates to no longer be available for additional testing for the OPAT clinician. As part of the OPAT evaluation and/or transition of care process, patients' microbiologic data should be evaluated to ensure all necessary information is obtained, especially if the patient is transferring from a different institution. For multidrug resistant or recurring infections, adding nonroutine susceptibilities should be considered, or communicating with clinical microbiology laboratory staff to release additional susceptibilities.

Certain barriers can be addressed by OPAT clinicians through pre-emptive collaboration with clinical microbiologists. This includes providing input on antimicrobial susceptibility testing (AST) panel design such as creating infection site-specific panels that include future oral agents, prespecifying certain AST released only by ID/OPAT clinicians' direct request (modified $\mathrm{CR}$ ), including narrative comments in the microbiology results section that informs on drug choice and dosing (e.g., defining susceptible-dose dependent (SDD) drug dose ranges), and implementing of AST rapid diagnostics/susceptibility (i.e., Accelerate Pheno). Additionally, electronic medical record clinical microbiologybased reports containing real time results of new positive cultures, broad range polymerase chain reactions (PCRs), serologies, and AST from OPAT/COpAT patients allow for same day interventions by OPAT clinicians as needed for new or different antimicrobials, streamlining/simplification of regimens, and addition of AST while samples are still available [26].

\section{Patient Adherence and Ease of Antimicrobial Administration}

In order for any antimicrobial to be effective, the patient must first take the antimicrobial. Less frequent dosing decreases the burden of effort on OPAT patients and their caregivers, particularly in the home setting. One study found that patients whose OPAT regimen was dosed once or twice 
daily were more likely to be adherent compared with patients whose antimicrobials were dosed more than twice daily [27••]. To facilitate less frequent dosing and to maximize chances of PK/PD target attainment, certain beta-lactams and vancomycin can be given as a continuous infusion (CI) [28-30]. The option for bolus or CI should be explored in advance as some patients may prefer multiple daily dosing, or insurance may not cover the necessary $\mathrm{CI}$ infusion pump devices [28]. The long-acting lipoglycopeptides oritavancin and dalbavancin are attractive options for patients requiring OPAT, since they are administered infrequently and do not require a PICC or midline, thereby increasing the chances of adherence $[31 \bullet, 32,33]$.

To facilitate less frequent dosing and ease of administration, a broader spectrum antimicrobial agent may be selected. Strategically choosing a broader spectrum agent is unlikely with inpatient stewardship teams, but patient convenience and ease of administration are often prioritized for OPAT. For example, daptomycin or ertapenem may be selected instead of vancomycin or other beta-lactams, because they are infused only once daily. When broader spectrum agents are used, efficacy, safety, and cost concerns must be addressed. One study suggested that daptomycin and ertapenem regimens may result in higher antimicrobial cost, but that drug purchase cost should be compared to the acute care admission costs saved by OPAT facilitating discharge [34]. They additionally found low rates of adverse events (12\% of readmissions) and C. difficile infection (7\%) with these broader spectrum agents. Likewise, in a small study comparing ceftriaxone, which is generally given once or twice daily, versus oxacillin or cefazolin, which are generally given three to six times daily, for methicillin-susceptible S. aureus (MSSA) bloodstream infections via OPAT, 90-day all-cause mortality, readmission due to MSSA bloodstream infection, microbiologic failure, and required antibiotic switches due to intolerance were all similar between the groups [35].

\section{Safety Monitoring}

Routine laboratory testing and safety monitoring are considered standard of care in OPAT. In the inpatient setting, serum laboratory testing is generally performed daily, and results are available within hours of order for the antimicrobial steward's review. In the outpatient setting, it is the OPAT team's responsibility to initiate laboratory orders and follow-up on results appropriately. Timely receipt of OPAT laboratory results can be challenging, and over half (58.5\%) of surveyed ID physicians reported this as a significant barrier to safe OPAT in one study [36••]. The non-availability of laboratory test results alone is independently associated with OPAT readmissions [37]. Opportunities exist for OPAT stewards, with these limi- tations in mind, to tailor frequency of laboratory testing and enhance safety monitoring plans for patients.

The risk of antibiotic-related adverse events increases with longer antibiotic duration in hospitalized patients [38]. Perhaps to a greater extent than their inpatient counterparts, OPAT/COpAT stewards must monitor closely for delayed-onset adverse drug reactions. Certain antibiotic-related adverse events occur on average two or more weeks after antibiotic initiation (e.g., linezolid-induced thrombocytopenia, beta-lactam-induced neutropenia) [39, 40]. Early post-discharge OPAT follow-up is necessary for timely detection and management of these events. Outpatient ID follow-up, including review and assessment of laboratory testing and adverse events within 2 weeks of hospital discharge, has been associated with reduced readmissions in patients receiving OPAT [41].

Many OPAT programs have adopted a once-weekly monitoring approach based on expert opinion and guideline recommendations [1]. Adjustments to a weekly schedule may be warranted based on antimicrobial regimen chosen, drug-drug interactions, and co-morbidities. More frequent laboratory testing may be needed in patients receiving aminoglycosides, while less frequent monitoring or no monitoring may be indicated when using longacting lipoglycopeptides. Adjustments in dosing schemes to facilitate laboratory testing and interpretation should be considered. If a CI vancomycin dosing scheme is employed, for example, blood samples can be drawn at any time after steady state has been achieved, and AUC24 can be estimated based on one level. Implementation of CI vancomycin can lead to reduction of 1) amount of care coordination required between OPAT programs, nursing facilities, and home infusion companies; 2) risk of inappropriately timed laboratory testing and delays in antibiotic dose adjustments; and 3) risk of antibiotic-related adverse events [30, 42].

For patients without regular clinical assessment in a nursing facility, there may be a role for expanded safety monitoring by the OPAT pharmacist. A study characterizing drugrelated problems experienced by patients on OPAT found that drug-drug interactions were the second most common type of problem [43]. Direct contact with a pharmacist post-hospital discharge can reduce medication-related problems and provide an opportunity for management of drug-drug interactions [44]. Pharmacist-conducted patient interviews may also be valuable in the identification of adverse events, as a high rate of discordance between clinician-reported and patientreported adverse events during OPAT has been described [45]. Lastly, clinicians are in a unique role to provide education, a goal important to patients, and their caregivers for the successful completion of OPAT at home [46••]. Unrealized opportunities may exist for comprehensive safety monitoring follow-up by a pharmacist beyond laboratory monitoring, 
including post-discharge medication reconciliation, assessment of medication adherence, management of drug-drug interactions, monitoring for patient-reported adverse events, and patient education. The successful use of telemedicine for OPAT follow-up and monitoring has previously been described and endorsed by the Infectious Diseases Society of America [47, 48]. Beyond the obvious advantages during the COVID-19 pandemic era, telemedicine may continue to play a role in the future of OPAT safety monitoring.

\section{OPAT Metrics}

Traditional antimicrobial stewardship metrics are difficult to implement in OPAT, since OPAT mainly consists of definitive therapy in non-acute care settings. Consensus is lacking around a set of accepted OPAT metrics. There is no separate accreditation for OPAT programs; however, they may qualify for Joint Commission outpatient antimicrobial stewardship accreditation [49] or ambulatory infusion center accreditation [50], depending on program structure. While this is an area of need for future consensus and development, some proposed initiatives and metrics are in Table 1.

National guidelines suggest potential OPAT program outcome measures, although these may not qualify as stewardship metrics. Patient clinical outcomes, readmission rates, OPAT therapy completion, and central line-related complications are commonly suggested [2, 51, 52] One group of researchers identified five core elements of inpatient antimicrobial stewardship which can pertain to OPAT: 1) antimicrobial use based on pathogen susceptibility, 2) duration of OPAT, 3) lack of $C$. difficile infection, 4) no emergency

Table 1 OPAT stewardship initiatives and program metrics

\begin{tabular}{|c|c|c|}
\hline Intervention & Potential benefits & Potential metric \\
\hline OPAT changed to COpAT $[59,60]$ & $\begin{array}{l}\text { Avoidance of requirement for PICC placement } \\
\text { and associated cost/risk of complications } \\
\text { Avoidance of need for home health, infusion } \\
\text { therapy center, or skilled nursing facility } \\
\text { services } \\
\text { Decrease laboratory monitoring requirements } \\
\text { Potential cost-savings to patient in the form of } \\
\text { direct antimicrobial costs } \\
\text { Favorable impacts on patient quality of life }\end{array}$ & $\begin{array}{l}\text { Line days avoid } \\
\text { Direct cost savings (PICC line, line mainte- } \\
\text { nance supplies, IV antibiotics, compounding } \\
\text { time, nursing time, etc.) } \\
\text { Patient satisfaction surveys }\end{array}$ \\
\hline Duration of therapy & $\begin{array}{l}\text { Decrease antimicrobial exposure/resistance } \\
\text { Decrease in adverse drug events, including } \\
\text { line-related }\end{array}$ & DOT vs clinical failures \\
\hline $\begin{array}{l}\text { Long-acting agents (dalbavancin, orita- } \\
\text { vancin) }[31 \bullet, 32,33]\end{array}$ & $\begin{array}{l}\text { Administered infrequently (often every } \\
7-14 \text { days) } \\
\text { Only require peripheral line (do not require a } \\
\text { PICC or midline) }\end{array}$ & $\begin{array}{l}\text { Line days saved/or OPAT days saved } \\
\text { Patient satisfaction surveys }\end{array}$ \\
\hline Continuous infusion antimicrobials $[2,42,61]$ & $\begin{array}{l}\text { Decrease admission to SNFs for administration } \\
\text { of IV antibiotics } \\
\text { Decrease in regimen changes for ease of } \\
\text { administration (e.g., daptomycin, ceftriax- } \\
\text { one) } \\
\text { Vancomycin specific benefit: } \\
\text { Decrease in inappropriately drawn vancomycin } \\
\text { TDM levels } \\
\text { Decrease in nephrotoxicity } \\
\text { Facilitates AUC:MIC monitoring }\end{array}$ & $\begin{array}{l}\text { Rate of antimicrobial regimen changes } \\
\text { Once daily antimicrobial utilization } \\
\text { Vancomycin time within goal } \\
\text { Rate of acute kidney injury }\end{array}$ \\
\hline Laboratory monitoring availability $[37,41]$ & Reduced health-care utilization during OPAT & Frequency of laboratory availability \\
\hline Outpatient clinical microbiology reporting & $\begin{array}{l}\text { Allows for real-time tailoring of antimicrobial } \\
\text { regimens based on microbiology results } \\
\text { Facilitates timely additional susceptibility test- } \\
\text { ing when needed }\end{array}$ & $\begin{array}{l}\text { Rate of de-escalation in the outpatient setting? } \\
\text { Time to appropriate therapy } \\
\text { Oral susceptibility reports available for each } \\
\text { organism }\end{array}$ \\
\hline Patient outcomes & $\begin{array}{l}\text { Annual tracking and benchmarking } \\
\text { Annual goal setting }\end{array}$ & $\begin{array}{l}\text { Time to outpatient follow up } \\
\text { OPAT completion rates } \\
\text { Adverse effects } \\
\text { Clostridioides difficile rates } \\
\text { 30-day ED/hospital readmission }\end{array}$ \\
\hline
\end{tabular}

Abbreviations: $A U C$ area under the concentration, COpAT complex outpatient antimicrobial therapy, DOT duration of therapy, ED emergency department, $I V$ intravenous, MIC minimum inhibitory concentration, OPAT outpatient parenteral antimicrobial therapy, $P I C C$ peripherally inserted central catheter, $S N F$ skilled nursing facility, TDM therapeutic drug monitoring 
department visit related to OPAT, and 5) no hospitalization related to OPAT [53]. It is important to differentiate between outcomes directly related to and influenced by OPAT versus other. Recent studies have shown implementing an OPAT "bundle" decreased 30-day readmission rates by half (13.0\% vs. $26.1 \%, P<0.01)$ [54]. Similarly, two studies developed risk prediction scores for 30-day readmission [55] and OPAT complications [56]. Potentially targeting these patient populations can improve OPAT program measures.

While two recent studies used RAND-modified Delphi procedures to create quality indicators for outpatient antimicrobial use, including OPAT, they did not overtly measure program outcomes or metrics $[57,58]$. Best practices such as multidisciplinary teams, patient and family education, medication storage, and communication strategies are stressed. However, some overlap with metrics included intravenous to oral switches, tracking treatment failure and adverse events, and rapid availability of laboratory values.

Access to a national database can lend to benchmarking against other similar institutions. The National Outcomes Registry System (NORS) database allows for this in the UK [52]; however, as of 2000, the USA is devoid of such a database [2]. Reviving and sustaining a database in the USA should be prioritized by regulatory and professional societies. Until such a database can be revived, institutions should create internal dashboards, identifying and tracking real-time data (current OPAT patients) and pertinent annual metrics. Similar to antimicrobial stewardship programs, these metrics should be reviewed annually and presented to leadership, with selection and work towards completing one goal annually.

\section{Conclusion}

Many advances and publications in the realm of OPAT and antimicrobial stewardship have been made in the last 5 years. In light of outpatient antimicrobial stewardship requirements by several US regulatory and accreditation bodies, the focus on OPAT stewardship will continue to increase. Creating a consensus on OPAT stewardship interventions and program metrics will be crucial for standardization and comparison. Until then, programs can consider applying OPAT to COpAT conversions, collaborating with microbiology laboratories on OPAT-related susceptibility algorithms, redesigning OPAT regimens upon transitions of care to ensure patient success, and employing appropriate safety and efficacy monitoring. Programs engaging in care for patients on OPAT/COpAT should be encouraged to publish on their experiences, in particular on stewardship metrics and program outcomes data.

\section{Compliance with Ethical Standards}

Conflict of Interest Drs. Childs-Kean, Khan, Rivera, Ryan, and Stevens have nothing to disclose. Dr. Mahoney reports personal fees from Tetraphase, personal fees from Spero, personal fees from Merck, personal fees from Paratek, personal fees from Qpex, personal fees and non-financial support from Cepheid, outside the submitted work.

Human and Animal Rights and Informed Consent This article does not contain any studies with human or animal subjects performed by any of the authors.

\section{References}

Papers of particular interest, published recently, have been highlighted as:

- Of importance

$\bullet$ Of major importance

1. Norris AH, Shrestha NK, Allison GM, Keller SC, Bhavan KP, Zurlo JJ, et al. 2018 Infectious Diseases Society of America Clinical Practice Guideline for the Management of Outpatient Parenteral Antimicrobial Therapy. Clin Infect Dis. 2019;68(1):e1-35.

2. Shah. Handbook of outpatient parenteral antimicrobial therapy for infectious diseases [Internet]. 3rd ed. Tarrytown: CRG Publishing and Infectious Diseases Society of America; 2016 [cited 2021 Jun 22]. Available from: https://www.idsociety.org/globalassets/ bb-complex-pages/idsa/opat-ehandbook/opat_epub_finalv2.pdf.

3.• Seaton RA, RitchieND, Robb F, Stewart L, White B, Vallance C. From 'OPAT' to 'COpAT':implications of the OVIVA study for ambulatory management of bone and jointinfection. J Antimicrob Chemother. 2019 Aug 1;74(8):2119-21. The first study to describe the concept of "COpAT".

4. Ohl CA, Luther VP. Antimicrobial stewardship for inpatient facilities. J Hosp Med. 2011;1(6 Suppl 1):S4-15.

5. Stevens RW, Estes L, Rivera C. Practical implementation of COVID-19 patient flags into an antimicrobial stewardship program's prospective review. Infect Control Hosp Epidemiol. 2020;41(9):1108-10.

6. Barlam TF, Cosgrove SE, Abbo LM, MacDougall C, Schuetz AN, Septimus EJ, et al. Implementing an antibiotic stewardship program: guidelines by the Infectious Diseases Society of America and the Society for Healthcare Epidemiology of America. Clin Infect Dis. 2016;62(10):e51-77.

7. Krein SL, Saint S, Trautner BW, Kuhn L, Colozzi J, Ratz D, et al. Patient-reported complications related to peripherally inserted central catheters: a multicentre prospective cohort study. BMJ Qual Saf. 2019;28(7):574-81.

8. Grau D, Clarivet B, Lotthé A, Bommart S, Parer S. Complications with peripherally inserted central catheters (PICCs) used in hospitalized patients and outpatients: a prospective cohort study. Antimicrob Resist Infect Control. 2017;6:18.

9. Dong Z, Connolly BL, Ungar WJ, Coyte PC. Cost analysis of peripherally inserted central catheter in pediatric patients. Int $\mathrm{J}$ Technol Assess Health Care. 2018;34(1):38-45.

10. Rivera CG, Mahoney MV, Stevens RW, Abu Saleh O. Out of sight but not out of mind: caring for patients receiving OPAT during the SARS-CoV-2 pandemic. Open Forum Infect Dis. 2020 Aug;7(8):ofaa309.

11. Krah NM, Bardsley T, Nelson R, Esquibel L, Crosby M, Byington $\mathrm{CL}$, et al. Economic burden of home antimicrobial therapy: OPAT versus oral therapy. Hosp Pediatr. 2019;9(4):234-40. 
12. - Marks M, Bell LCK, Jones I, Rampling T, Kranzer K, MorrisJones S, et al. Clinical and economic impact of implementing OVIVA criteria on patients with bone and joint infections in outpatient parenteral antimicrobial therapy. Clin Infect Dis Off Publ Infect Dis Soc Am. 2020 Jun 24;71(1):207-10. A study investigating how many patients discharged with OPAT could have been treated with oral antibiotics instead.

13. Azamgarhi T, Shah A, Warren S. Clinical experience of implementing oral versus intravenous antibiotics (OVIVA) in specialist orthopedic hospital. Clin Infect Dis 2020;ciaa985. doi: https:// doi.org/10.1093/cid/ciaa985.

14. Vollmer NJ, Rivera CG, Stevens RW, Oravec CP, Mara KC, Suh $\mathrm{GA}$, et al. Safety and tolerability of fluoroquinolones in patients with staphylococcal periprosthetic joint infections. Clin Infect Dis Off Publ Infect Dis Soc Am. 2021 Feb 19; ciab145.

15. Core elements of hospital antibiotic stewardship programs I antibiotic use I CDC [Internet]. 2021 [cited 2021 Jun 22]. Available from: https://www.cdc.gov/antibiotic-use/core-elements/hospital.html.

16. Sawyer RG, Claridge JA, Nathens AB, Rotstein OD, Duane TM, Evans HL, et al. Trial of short-course antimicrobial therapy for intraabdominal infection. N Engl J Med. 2015;372(21):1996-2005.

17. Yahav D, Franceschini E, Koppel F, Turjeman A, Babich T, Bitterman R, et al. Seven versus 14 days of antibiotic therapy for uncomplicated gram-negative bacteremia: a noninferiority randomized controlled trial. Clin Infect Dis Off Publ Infect Dis Soc Am. 2019;69(7):1091-8.

18. Gariani K, Pham T-T, Kressmann B, Jornayvaz FR, Gastaldi G, Stafylakis D, et al. Three versus six weeks of antibiotic therapy for diabetic foot osteomyelitis: a prospective, randomized, noninferiority pilot trial. Clin Infect Dis Off Publ Infect Dis Soc Am. 2020 Nov 26; ciaa1758.

19. Gjika E, Beaulieu J-Y, Vakalopoulos K, Gauthier M, Bouvet C, Gonzalez A, et al. Two weeks versus four weeks of antibiotic therapy after surgical drainage for native joint bacterial arthritis: a prospective, randomised, non-inferiority trial. Ann Rheum Dis. 2019;78(8):1114-21.

20. Bernard L, Dinh A, Ghout I, Simo D, Zeller V, Issartel B, et al. Antibiotic treatment for 6 weeks versus 12 weeks in patients with pyogenic vertebral osteomyelitis: an open-label, noninferiority, randomised, controlled trial. Lancet Lond Engl. 2015;385(9971):875-82.

21. Palit J, Cole J, Durojaiye OC. Clinical and operational factors associated with treatment duration for cellulitis in outpatient parenteral antimicrobial therapy (OPAT). Diagn Microbiol Infect Dis. $2021 ; 100(1): 115305$.

22. Liao S, Rhodes J, Jandarov R, DeVore Z, Sopirala MM. Out of sight-out of mind: impact of cascade reporting on antimicrobial usage. Open Forum Infect Dis. 2020 8;7(2):ofaa002. Using cascade reporting for ceftriaxone decreased broad-spectrum antibiotic use for Ecoli and Klebsiella.

23. Al-Tawfiq JA, Momattin H, Al-Habboubi F, Dancer SJ. Restrictive reporting of selected antimicrobial susceptibilities influences clinical prescribing. J Infect Public Health. 2015;8(3):234-41.

24. Johnson LS, Patel D, King EA, Maslow JN. Impact of microbiology cascade reporting on antibiotic de-escalation in cefazolinsusceptible Gram-negative bacteremia. Eur J Clin Microbiol Infect Dis Off Publ Eur Soc Clin Microbiol. 2016;35(7):1151-7.

25. Liao S, Rhodes J, Jandarov, DeVore Z, Sopirala MM. Out of sight - out of mind: impact of cascade reporting on antimicrobial usage. Open Forum Infect Dis 2020; ofaa002 doi:https://doi.org/ 10.1093/ofid/ofaa002.

26. Rivera CG, Zeuli J. Outpatient ID pharmacists: OPAT and beyond. poster presented at American College of Clinical Pharmacy annual meeting; 2019 Oct 26.
27.• HamadY, Dodda S, Frank A, Beggs J, Sleckman C, Kleinschmidt $\mathrm{G}$, et al. Perspectives ofpatients on outpatient parenteral antimicrobial therapy: experiences andadherence. Open Forum Infect Dis [Internet]. 2020 Jun 1 [cited 2021 Jun22];7(6). Available from: https://doi.org/10.1093/ofid/ofaa205An Important survey of patients receiving OPAT and factors that lead to non-adherance.

28. Steffens E, Quintens C, Derdelinckx I, Peetermans WE, Van Eldere J, Spriet I, et al. Outpatient parenteral antimicrobial therapy and antibiotic stewardship: opponents or teammates? Infection. 2019;47(2):169-81.

29. Jones BM, Huelfer K, Bland CM. Clinical and safety evaluation of continuously infused Ceftolozane/Tazobactam in the outpatient setting. Open Forum Infect Dis. 2020 Feb;7(2):ofaa014

30. Shakeraneh P, Fazili T, Wang D, Gilotra T, Steele JM, Seabury RW, et al. Nephrotoxicity risk and clinical effectiveness of continuous versus intermittent infusion vancomycin among patients in an outpatient parenteral antimicrobial therapy program. Pharmacotherapy. 2020;40(4):357-62.

31. Redell M, Sierra-Hoffman M, Assi M, Bochan M, Chansolme D, Gandhi A, et al. The CHROME study, a real-world experience of single- and multiple-dose Oritavancin for treatment of gram-positive infections. Open Forum Infect Dis. 2019 Nov;6(11):ofz479. Large-scale, real-world use of Oritavancin for Gram-positive infections.

32. Morata L, Cobo J, Fernández-Sampedro M, Guisado Vasco P, Ruano E, Lora-Tamayo J, et al. Safety and efficacy of prolonged use of dalbavancin in bone and joint infections. Antimicrob Agents Chemother. 2019;63(5):e02280-e2318.

33. Buzón Martín L, Mora Fernández M, Perales Ruiz JM, Ortega Lafont M, Álvarez Paredes L, Morán Rodríguez MA, et al. Dalbavancin for treating prosthetic joint infections caused by Gram-positive bacteria: a proposal for a low dose strategy. A retrospective cohort study. Rev Espanola Quimioter Publicacion Of Soc Espanola Quimioter. 2019 Dec;32(6):532-8.

34. Britt RS, LaSalvia MT, Padival S, Patel P, McCoy C, Mahoney MV. Evaluation of inpatient antimicrobial regimens for readmitted outpatient parenteral antimicrobial therapy patients receiving Daptomycin or Ertapenem for ease of administration. Open Forum Infect Dis. 2019 Dec;6(12):ofz496.

35. amad Y, Connor L, Bailey TC, George IA. Outcomes of outpatient parenteral antimicrobial therapy with ceftriaxone for methicillin-susceptible Staphylococcus aureus bloodstream infections-a single-center observational study. Open Forum Infect Dis. 2020 Sep;7(9):ofaa341.

36. • Hamad Y, Lane MA, Beekmann SE, Polgreen PM, Keller SC. Perspectives of United States-based infectious diseases physicians on outpatient parenteral antimicrobial therapy practice. Open Forum Infect Dis. 2019 Oct 1;6(10):ofz363. An important survey of ID physicians regarding composition of OPAT programs.

37. Huck D, Ginsberg JP, Gordon SM, Nowacki AS, Rehm SJ, Shrestha NK. Association of laboratory test result availability and rehospitalizations in an outpatient parenteral antimicrobial therapy programme. J Antimicrob Chemother. 2014;69(1):228-33.

38. Tamma PD, Avdic E, Li DX, Dzintars K, Cosgrove SE. Association of adverse events with antibiotic use in hospitalized patients. JAMA Intern Med. 2017;177(9):1308.

39. Giunio-Zorkin N, Brown G. Real-life frequency of new-onset thrombocytopenia during linezolid treatment. Can J Hosp Pharm. 2019;72(2):133-8.

40. Cimino C, Allos BM, Phillips EJ. A Review of $\beta$-Lactamassociated neutropenia and implications for cross-reactivity. Ann Pharmacother. 2021;55(8):1037-49. 
41. Saini E, Ali M, Du P, Crook T, Zurlo J. Early infectious disease outpatient follow-up of outpatient parenteral antimicrobial therapy patients reduces 30-day readmission. Clin Infect Dis Off Publ Infect Dis Soc Am. 2019 Aug 16;69(5):865-8. *A potential OPAT quality metric, early outpatient follow-up (within 14 days) was linked with lower 30-day readmission rates.

42. Rybak MJ, Le J, Lodise TP, Levine DP, Bradley JS, Liu C, et al. Therapeutic monitoring of vancomycin for serious methicillinresistant Staphylococcus aureus infections: a revised consensus guideline and review by the American Society of Health-System Pharmacists, the Infectious Diseases Society of America, the Pediatric Infectious Diseases Society, and the Society of Infectious Diseases Pharmacists. Am J Health-Syst Pharm AJHP Off J Am Soc Health-Syst Pharm. 2020;77(11):835-64.

43. Hale CM, Steele JM, Seabury RW, Miller CD. Characterization of drug-related problems occurring in patients receiving outpatient antimicrobial therapy. J Pharm Pract. 2017;30(6):600-5.

44. Daliri S, Hugtenburg JG, Riet G ter, Bemt BJF van den, Buurman BM, Reimer WJMS op, et al. The effect of a pharmacy-led transitional care program on medication-related problems post-discharge: a before-after prospective study. PLOS ONE. 2019 Mar 12;14(3):e0213593.

45. Keller SC, Williams D, Gavgani M, Hirsch D, Adamovich J, Hohl D, et al. Rates of and risk factors for adverse drug events in outpatient parenteral antimicrobial therapy. Clin Infect Dis. 2018;66(1):11-9.

46. $\bullet$ Keller SC, Cosgrove SE, Arbaje AI, Chang RH, Krosche A, Williams D, et al. It's complicated: patient and informal caregiver performance of outpatient parenteralantimicrobial therapy-related tasks. Am J Med Qual Off J Am Coll Med Qual. 2020;35(2):13346. A survey of patient receiving OPAT which identifies key areas to improve patient understanding and adherence.

47. Tan SJ, Ingram PR, Rothnie AJ, Whitmore TJ, Robinson JO, Hatch $\mathrm{JB}$, et al. Successful outpatient parenteral antibiotic therapy delivery via telemedicine. J Antimicrob Chemother. 2017;72(10):2898-901.

48. Young JD, Abdel-Massih R, Herchline T, McCurdy L, Moyer KJ, Scott JD, et al. Infectious Diseases Society of America Position Statement on Telehealth and Telemedicine as Applied to the Practice of Infectious Diseases. Clin Infect Dis Off Publ Infect Dis Soc Am. 2019;68(9):1437-43.

49. The Joint Commission. R3 report issue 23: antimicrobial stewardship in ambulatory health care. Available at: https://www.jointcommission. org/standards/r3-report/r3-report-issue-23-antimicrobial-stewardshipin-ambulatory-health-care/ Accessed 9/25/21.

50. Authors No. Joint Commission Now Accredits Ambulatory Care Infusion Centers. Jt Comm Perspect. 1995;15:15-6.

51. Tice AD, Rehm SJ, Dalovisio JR, Bradley JS, Martinelli LP, Graham DR, et al. Practice guidelines for outpatient parenteral antimicrobial therapy. IDSA guidelines. Clin Infect Dis Off Publ Infect Dis Soc Am. 2004 Jun 15;38(12):1651-72.
52. ALN Chapman S Patel C Horner H Green A Guleri S Hedderwick Updated good practice recommendations for outpatient parenteral antimicrobial therapy (OPAT) in adults and children in the UK. JAC-Antimicrob Resist [Internet]. et al 2019 Sep 1 [cited 2021 Jun 22];1(2) Available from: https://doi.org/10.1093/jacamr/dlz026.

53. Nathan RV, Adams JS, Dretler RH, Luu Q, Metzger BS, Schroeder $\mathrm{CP}$, et al. Successful establishment of an antimicrobial stewardship program (ASP) for outpatient parenteral antimicrobial therapy (OPAT). Open Forum Infect Dis. 2017;4(Suppl 1):S259.

54. Madaline T, Nori P, Mowrey W, Zukowski E, Gohil S, Sarwar U, et al. Bundle in the Bronx: impact of a transition-of-care outpatient parenteral antibiotic therapy bundle on all-cause 30-day hospital readmissions. Open Forum Infect Dis. 2017;4(2):ofx097.

55. Durojaiye OC, Kritsotakis EI, Johnston P, Kenny T, Ntziora F, Cartwright K. Developing a risk prediction model for 30-day unplanned hospitalization in patients receiving outpatient parenteral antimicrobial therapy. Clin Microbiol Infect Off Publ Eur Soc Clin Microbiol Infect Dis. 2019;25(7):905.e1-905.e7.

56. Keller SC, Wang N-Y, Salinas A, Williams D, Townsend J, Cosgrove SE. Which patients discharged to home-based outpatient parenteral antimicrobial therapy are at high risk of adverse outcomes? Open Forum Infect Dis. 2020 Jun; 7(6):ofaa178.

57. Le Maréchal M, Tebano G, Monnier AA, Adriaenssens N, Gyssens IC, Huttner B, et al. Quality indicators assessing antibiotic use in the outpatient setting: a systematic review followed by an international multidisciplinary consensus procedure. J Antimicrob Chemother. 2018 Jun 1;73(suppl_6):vi40-9.

58. Berrevoets MAH, Ten Oever J, Oerlemans AJM, Kullberg BJ, Hulscher ME, Schouten JA. Quality indicators for appropriate outpatient parenteral antimicrobial therapy in adults: a systematic review and RAND-modified Delphi procedure. Clin Infect Dis Off Publ Infect Dis Soc Am. 2020;70(6):1075-82.

59. Li H-K, Rombach I, Zambellas R, Walker AS, McNally MA, Atkins BL, et al. Oral versus intravenous antibiotics for bone and joint infection. N Engl J Med. 2019;380(5):425-36.

60. Iversen K, Ihlemann N, Gill SU, Madsen T, Elming H, Jensen $\mathrm{KT}$, et al. Partial oral versus intravenous antibiotic treatment of endocarditis. N Engl J Med. 2019;380(5):415-24.

61. Voumard R, Gardiol C, André P, Arensdorff L, Cochet C, BoillatBlanco N, et al. Efficacy and safety of continuous infusions with elastomeric pumps for outpatient parenteral antimicrobial therapy (OPAT): an observational study. J Antimicrob Chemother. 2018;73(9):2540-5.

Publisher's Note Springer Nature remains neutral with regard to jurisdictional claims in published maps and institutional affiliations. 